\title{
Formal reconciliatory dialogue based on shift from forward to backward deliberation ${ }^{\dagger}$
}

\author{
Hiroyuki Kido ${ }^{\mathrm{a} *}$ and Federico Cerutti ${ }^{\mathrm{b}}$ \\ ${ }^{a}$ School of Engineering, University of Tokyo, 7-3-1 Hongo, Bunkyo-ku, Tokyo 113-8656, Japan; ${ }^{b}$ School of \\ Computer Science \& Informatics, Cardiff University, Queen's Buildings, 5 The Parade, Cardiff CF24 3AA, \\ $U K$
}

(Received 17 March 2015; accepted 7 December 2015)

\begin{abstract}
Desire conflicts arise in several real-world contexts. In this paper, we propose a mixed deliberation dialogue for reconciliation. A mixed deliberation dialogue is defined as a combination of forward and backward deliberation dialogues with respective goals which are subordinate and superordinate desires of a given desire. This research and the introduction of mixed deliberation dialogue have been motivated by Kowalski and Toni's reconciliatory scenario. We show that an instantiation of a mixed deliberation dialogue implements key parts of Kowalski and Toni's reconciliatory solution. We also prove the correctness of mixed deliberation dialogues.
\end{abstract}

Keywords: formal dialogue; deliberation; dialogue shift; reconciliation; defeasible inferencerules

\section{Introduction}

Dialogue theory encompasses various types of descriptive and formal studies, aimed at various purposes, on the structure of dialogues (van Eemeren, Grootendorst, and Henkemans, 1996). Hintikka's game-theoretic semantics (1968) and Lorenzen's dialogue logic (1961) explore semantics of language. Hamblin's formal dialectics (1970) explores descriptive or formal dialogue systems. Because formal dialogue systems can give agents rational interaction and computation mechanisms under uncertain, incomplete, inconsistent, subjective, and distributed information, they have received attention from researchers working on formal argumentation (Fan and Toni, 2012; Kok, Meyer, Prakken, and Vreeswijk, 2010; Prakken, 2006, 2005; Wells and Reed, 2006).

However, little work has been done for dialogue systems for reconciling conflict not only by searching for means of satisfying either all or part of given desires, but also by searching for means for satisfying their underlying desires behind the given ones. Kowalski and Toni (1994) first presented arguments for the necessity of reconciliation in the context of argumentation.

What is interesting about their scenario is that neither the generalised goal nor reconciliatory solution is obtainable merely by just choosing one of the given alternatives based on utilities or preferences, that is, quantitative measures, but their scenario requires some sort of qualitative measure to shift to an underlying desire. Based on the scenario, they outlined what a generalised goal and a reconciliatory solution are. However, an open question is how one should have a dialogue to reach the generalised goal and the reconciliatory solution. These observations motivate us to formalise reconciliatory dialogues as consisting of forward and backward deliberation dialogues.

\footnotetext{
*Corresponding author. Email: kido@sys.t.u-tokyo.ac.jp

${ }^{\dagger}$ This paper is a revised full version of the conference paper (Kido and Cerutti, 2014).
} 
This paper contributes to the state of the art of studies of formal dialogue and argumentation by handling the processes leading from conflict detection to justification of reconciliation in terms of a series of dialogues. Particularly, this paper gives underlying dialogue and inference principles behind reconciliation. Furthermore, we address Kowalski and Toni's academically acknowledged scenario that cannot be solved simply by taking advantage of utilities or preferences assumed in many formal dialogue-based and argumentation-based approaches.

This paper is organised as follows. Section 2 motivates the research referring to Kowalski and Toni's scenario: a running example is used throughout the paper to describe our proposal. Section 3 presents some preliminary notions that are used widely to define dialogue goals in Section 4 and dialogue protocols in Section 5. In Section 6, we proved the correctness of the dialogues with respect to their goals. Section 7 discusses related work, and Section 8 concludes the paper. Proofs of results are given in Appendix.

\section{Motivation}

We consider the following realistic reconciliatory scenario demonstrating the importance of goal generalisation.

Example 1 (Kowalski and Toni, 1994): In a recent head-of-sections committee meeting in our Department, we discussed the composition of a new resources committee. Two conflicting arguments were put forward. The Director of Administration argued that, in the interests of efficiency, the members of the new committee should consist of himself and the other principal administrative officers of the Department. The Director of Research argued, in opposition to him, that, in the interests of democracy, the committee should also contain members elected by the Department.

During the course of the discussion, it became clear that the two sides were focusing on different assumptions about the purpose of the new committee: the Director of Administration on its purely administrative function, and the Director of Research on its presumedpolicy-making nature. These two assumptions could be viewed as conflicting solutions to the more general goals of deciding, on the one hand, which group should administer resources, and on the other hand, which group should make policy about resources.

By focusing on the more general goals, it was possible to identify a new solution which was acceptable to both parties: the resources committee will administer resources, whereas the head-of-sections committee will make policy about resources. In the interests of efficiency, the members of the resources committee will consist of administrative officers only. In the interests of democracy, the head-of-sections committee will represent the views and interests of the various Department sections on matters concerning policy about the allocation of resources.

In this scenario, neither a generalised goal nor a reconciliatory solution is obtainable merely by just choosing a given alternative based on utility or preference. An open question is how one should have a dialogue to reach the generalised goal and the reconciliatory solution.

Therefore, we formalise reconciliatory dialogues as consisting of forward and backward deliberation dialogues. We show how shifting between forward and backward deliberation dialogue highlights the linkage with more general goals. On the one hand, the Director of Administration desires efficiency. On the other hand, the Director of Research wants to promote democracy via voting although this demotes efficiency-forward deliberation. Then, because one outcome of adopting democracy is fairness, and because there is no reason in favour of not desiring fairness, we can consider it a 'desirable' outcome of the democracy-backward deliberation. Finally, shifting back to a forward deliberation dialogue, the two more general goals become evident, because efficiency will be improved if the resources committee comprises administrative 


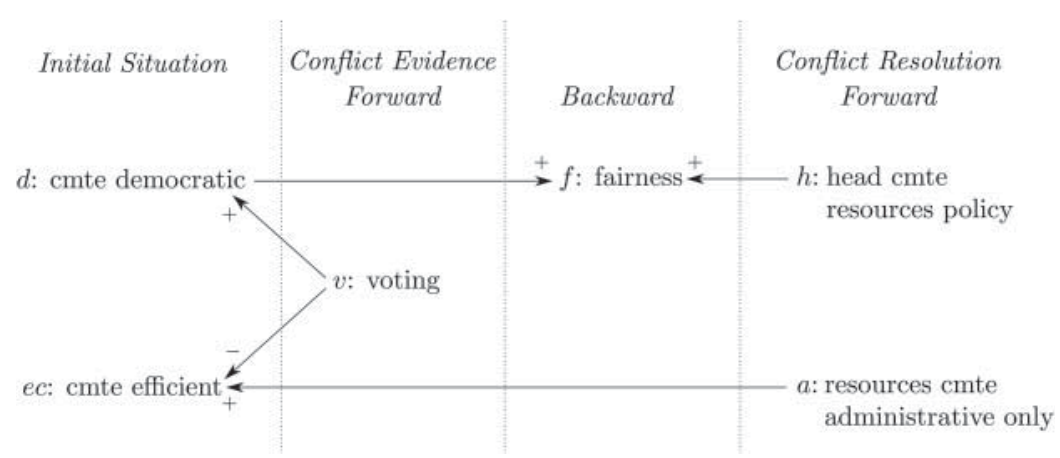

Figure 1. Rough causal relations behind Kowalski and Toni’s reconciliatory scenario.

officers only, and fairness will be ensured if the head committee will take care of making policies related to resources.

In Figure 1, we show rough causal relations behind their scenario with some additional information where nodes, respectively, represent statements and arrows, from node $x$ to node $y$, attached with + (resp. - ) represent $x$ promotes (resp. demotes) $y$. For the discussion, we expand Kowalski and Toni's scenario.

Example 1 (continued): According to Figure 1:

- $d$ represents 'the resources committee is democratic';

- $e c$ represents 'the resources committee is efficient';

- $v$ represents 'the resources committee is elected by vote';

- $f$ represents 'the policy is fair';

- $h$ represents 'the head committee makes policy about resources';

- $a$ represents 'the resources committee is composed by administrative officers only'.

\section{Preliminaries}

We use Dung's theory of acceptability semantics (1995) that reformulates consequence notions of nonmonotonic logics. The semantics is defined on a pair $A F=\langle A R$, attacks $\rangle$, called an abstract argumentation framework, where $A R$ is a set of arguments and attacks is a binary relation on $A R$, that is, attacks $\subseteq A R \times A R$.

Definition 1 (Dung, 1995): Let $A F=\langle A R$, attacks $\rangle$ be an abstract argumentation framework, $S \subseteq A R$ and $a \in A R$.

- $S$ is conflict-free iff no $a, b \in S$ exists such that a attacks $b$, that is,$(a, b) \notin$ attacks.

- a is acceptable with respect to $S$ iff, for all $b \in A R$, if $b$ attacks a then there is $c \in S$ such that $c$ attacks $b$.

- The characteristic function, $F_{A F}: \operatorname{Pow}(A R) \rightarrow \operatorname{Pow}(A R)$, is defined as $F_{A F}(S)=\{a \in$ $A R \mid$ a is acceptable with respect to $S\}$.

- $S$ is the grounded extension iff it is the least fixed point of $F_{A F}$.

Given an argumentation framework $A F=\langle A R$, attacks $\rangle$, we say that an argument $a \in A R$ is justified in $A F$ iff $a$ is in the grounded extension of $A F$, and overruled otherwise. 
In the following, we consider argumentation frameworks instantiated from $\mathcal{L}_{0}$, a language of modal propositional logic with single modal operator $D$ representing the operator 'it is desirable that'. $\mathcal{L}_{0}$ is closed under truth-functional operations. Consequently, if $a \in \mathcal{L}_{0}$, then $\neg a \in \mathcal{L}_{0}$; if $a, b \in \mathcal{L}_{0}$, then $a \vee b, a \wedge b, a \rightarrow b \in \mathcal{L}_{0}$; etc. $\mathcal{L}_{0}$ conforms to the axiomatic system KD in which $D(a \rightarrow b) \rightarrow D a \rightarrow D b$ and $D a \rightarrow \neg D \neg a$ are axioms. Also, $\mathcal{L}_{1}$ consists of so-called defeasible conditionals, or defaults. They commonly have the forms ' $a \Rightarrow b$ ', where $a$ is a conjunction of literals, that is, atomic propositions or their negation, in $\mathcal{L}_{0}$ and $b$ is a literal in $\mathcal{L}_{0}$, and mean that if $a$ is the case, then $b$ is normally the case. Operator $D$ is assumed not to appear in defeasible conditionals. We assume a fixed, but arbitrary theory $T \subseteq \mathcal{L}_{0} \cup \mathcal{L}_{1}$.

Example 1 (continued): To illustrate our proposal, we consider the following additional propositions:

- $e$ represents 'the policy is effective';

- $m$ represents 'the resources committee makes policy about resources';

- $r$ represents 'the head-of-sections committee represents various views of interests'.

Moreover, let us consider the following rules, which provide the casual relations depicted in Figure 1:

$$
\begin{gathered}
v \Rightarrow d ; \quad v \Rightarrow \neg e c ; \quad a \Rightarrow e c ; \quad d \wedge m \Rightarrow f ; \\
d \wedge m \Rightarrow \neg e ; \quad \text { ec } \wedge m \Rightarrow e ; \quad h \wedge r \Rightarrow f .
\end{gathered}
$$

Any rule of inference that is not valid with respect to modal logic KD is called a defeasible inference rule, represented by $\rightsquigarrow$. More precisely, a rule of inference is not valid if it can derive a formula that is not a theorem in KD. Intuitively, an inference is not valid if it is not deductive in terms of KD. We use letters $a, b, c, \ldots$ of the alphabet to represent literals in $\mathcal{L}_{0} \cup \mathcal{L}_{1}$, lower case Greek letters $\alpha, \beta, \gamma, \ldots$ to represent their metavariables of them and $A, B, C, \ldots$ to represent their sequences.

Definition 2 (Bench-Capon and Prakken, 2006): Positive forward practical syllogism, denoted by PFPS, and negative forward practical syllogism, denoted by NFPS, are defined as follows, respectively ${ }^{1}$ :

$$
\begin{aligned}
& \text { PFPS :D } \alpha, \gamma, \beta \wedge \gamma \Rightarrow \alpha \rightsquigarrow D \beta ; \\
& \text { NFPS :D } \alpha, \gamma, \beta \wedge \gamma \Rightarrow \neg \alpha \rightsquigarrow D \neg \beta .
\end{aligned}
$$

The positive one states intuitively that if one believes that $\alpha$ is desirable (e.g. 'democratic cmte' $d$ ), $\gamma$ is the case and if $\beta$ (e.g. 'voting' $v$ ) is realised under $\gamma$ is the case then $\alpha$ is satisfied, then one defeasibly concludes that $\beta$ is desirable $(D d, v \Rightarrow d \rightsquigarrow D v)$. The negative one, however, concludes that $\beta$ is undesirable from a different premise stating that if $\beta$ is realised under $\gamma$ is the case, then $\alpha$ is frustrated. We describe them as FPS without distinction. We often silently use abbreviated forms $D \alpha, \beta \Rightarrow \alpha \rightsquigarrow D \beta$ and $D \alpha, \beta \Rightarrow \neg \alpha \rightsquigarrow D \neg \beta$.

Definition 3: Let $\Sigma \subseteq T, p_{i} \in \mathcal{L}_{0} \cup \mathcal{L}_{1}$. A sequence $A=p_{1}, p_{2}, \ldots, p_{n}$ is an argument from $\Sigma$ to $p_{n}$ iff (1) for all $i(1 \leq i \leq n), p_{i} \in \Sigma$ (base case) or $p_{i}$ is derived from preceding formulae $p_{j}(j<i)$ by application of a rule of inference, and (2) for all $i(1 \leq i \leq n)$, the sequence obtained by eliminating $p_{i}$ from A does not satisfy (1).

The first condition states that an argument is a derivation from $\Sigma$ using strict and defeasible inference rules. The second assures that an argument consists of a minimal number of formulae. 
As described herein $\Sigma \vdash_{x} p_{n}$ denotes an argument from $\Sigma$ to $p_{n}$ where rules of inference are restricted to only $x$. For example, $\Sigma \vdash_{F P S} D a$ represents that $D a$ is derived from $\Sigma$ by application of only positive or negative forward practical syllogisms zero or more times.

\section{Formal goals of deliberation dialogues}

\subsection{Superordinate and subordinate desires}

This section provides formal definitions of dialogue goals. Given a desirable outcome or desire, we use the term superordinate desire to refer to a desire such that, once it is assumed, it can be a rationale for desiring a given desire, but it cannot be a rationale for not desiring a given desire. Based on the recognition that the practical syllogisms give fundamental inference mechanisms for practical reasoning, we give a formal definition of superordinate desires as follows.

Definition 4: Let $D g, D h \in \mathcal{L}_{0}$. Dh is a superordinate desire of $D g$ in $T$ iff $\Sigma_{1} \subseteq T$ exists such that $\Sigma_{1} \cup\{D h\} \vdash_{F P S} D g$ and no $\Sigma_{2} \subseteq T$ exists such that $\Sigma_{2} \cup\{D h\} \vdash_{F P S} D \neg t$, for all $D t \in T \cup$ $\{D g\}$.

Definition 4 states that a superordinate desire $D h$ derives $D g$ by application of only forward practical syllogisms $\vdash_{F P S}$, but no negation of any desire in $T \cup\{D g\}$ can be derived by their application. It states that $h$ would be desirable because, once $D h$ is assumed, it can be a rationale for $D g$. Definition 4 is weaker than the definition replacing $\Sigma$ to $T$ because it permits $T \vdash_{F P S} D g$, that is, the situation in which $D g$ is derived from $T$ without using $D h$. Moreover, in general, a superordinate desire is not intrinsically a desire existing in theory $T$, but it is derived by defeasible inferences.

Example 1 (continued): $D f$ is a superordinate desire of $D d$ in $T=\{d \wedge m \Rightarrow f, d \wedge m \Rightarrow$ $\neg e, e c \wedge m \Rightarrow e, D e c, m\}$. However, $D \neg e$ is not because of the following reason. Once $D \neg e$ is assumed, $D d$ is derived from $\Sigma_{1}=\{d \wedge m \Rightarrow \neg e, m\}(\subseteq T)$, that is, $\Sigma_{1} \cup\{D \neg e\} \vdash_{F P S} D d$. However, once $D \neg e$ is assumed, $D \neg e c$ is derived from $\Sigma_{2}=\{e c \wedge m \Rightarrow e, m\}(\subseteq T)$, that is, $\Sigma_{2} \cup\{D \neg e\} \vdash_{F P S} D \neg e c$. Figure 2 shows rough causal relations behind this superordinate desire. One can see that $D d$ is derived from the assumption $D f$ using FPS. Although it can also be derived from the assumption $D \neg e$, the assumption results in a derivation of $D \neg e c$ that conflicts with $D e c \in T$.

We introduce a notion of subordinate desires. Given a desire, we use the term subordinate desire to refer to a desire such that if once it is realised then it satisfies at least one of the given desires, but it frustrates no desire in them. Namely, a subordinate desire of a desire is desirable as a means of satisfying the desire, but not as a means of frustrating any other desire.

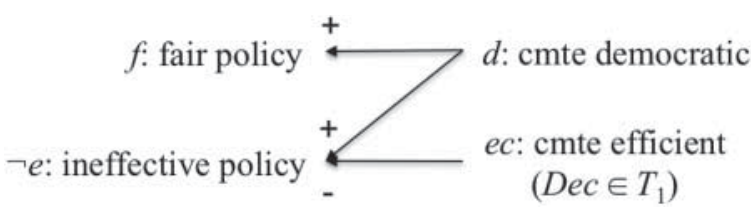

Figure 2. Rough causal relations behind the superordinate desire in Example 1. 


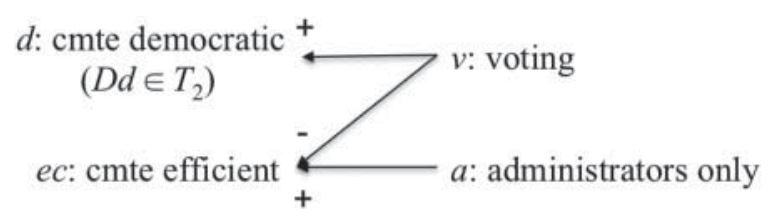

Figure 3. Rough causal relations behind the subordinate desire in Example 1.

Definition 5: Let $D g, D h \in \mathcal{L}_{0}$. Dg is a subordinate desire of Dh in T iff there is $\Sigma_{1} \subseteq T$ such that $\Sigma_{1} \cup\{D h\} \vdash_{F P S} D g$ and there is no $\Sigma_{2} \subseteq T$ such that $\Sigma_{2} \cup\{D t\} \vdash_{F P S} D \neg g$, for all Dt $\in$ $T \cup\{D h\}$.

Definition 5 states that the subordinate desire $D g$ is derived by application only of forward practical syllogisms, but no desire in $T \cup\{D h\}$ derives its negation $D \neg g$ by application of them. It states that $g$ is desirable as a means for satisfying $h$ without frustrating any desire in $T \cup\{D h\}$. As with superordinate desires, Definition 5 is weaker than the definition replacing $\Sigma$ to $T$. In general, a subordinate desire is not intrinsically a desire existing in theory $T$.

Example 1 (continued): $D a$ is a subordinate desire of Dec in $T=\{v \Rightarrow d, v \Rightarrow \neg e c, a \Rightarrow$ $e c, D d\}$. However, $D \neg v$ is not because, given $\Sigma_{1}=\{v \Rightarrow \neg e c\}$, although $\Sigma_{1} \cup\{D e c\} \vdash_{F P S} D \neg v$, but given $\Sigma_{2}=\{v \Rightarrow d\}, \Sigma_{2} \cup\{D d\} \vdash_{F P S} D v$. Figure 3 shows rough causal relations behind this subordinate desire. It might be readily apparent that $D a$ is derived from the assumption $D e c$ using $F P S$. Although $D \neg v$ can also be derived from $D e c$, it conflicts with $D v$ derived from $D d \in T$ using FPS.

\subsection{Reconciliatory desires as a combination of superordinate and subordinate desires}

Finally, we introduce the notion of reconciliatory desires defined by combining superordinate desires and subordinate desires.

Definition 6: Let $D g, D h \in \mathcal{L}_{0}$. Dg is a reconciliatory desire of $D h$ in $T$ iff $D i \in \mathcal{L}_{0}$ exists such that $\mathrm{Di}$ is a superordinate desire of $\mathrm{Dh}$ in $\mathrm{T}$ and $\mathrm{Dg}$ is a subordinate desire of $\mathrm{Di}$ in $T$.

Definition 6 states that $g$ is desirable because it does not frustrate any desire in $T \cup\{D i\}$, but satisfies $i$. Here, $i$ is regarded as desirable because, once it is assumed, it gives a rationale for desiring $h$. Note that subordinate and superordinate desires are both special cases of reconciliatory desires.

Example 1 (continued): $T=\{d \wedge m \Rightarrow f, d \wedge m \Rightarrow \neg e, e c \wedge m \Rightarrow e, m, D e c, h \wedge r \Rightarrow f, r\} . D h$ is a reconciliatory desire of $D d$ in $T$ because $D f$ is a superordinate desire of $D d$, and $D h$ is a subordinate desire of $D f$.

\subsection{Backward practical syllogisms}

We introduce a backward version of the practical syllogism. This inference pattern, differently from the traditional forward practical syllogism, represents the result of a critical thinking approach. Indeed, presuming that $\alpha$ is a desirable outcome $(D \alpha)$, and assuming it is true that $\alpha \Rightarrow \beta$. Therefore, if $\alpha$ is accepted as desirable, then $\beta$, because it is a material implication deriving from $\alpha$, should be considered. What the backward practical syllogism is doing here is to suggest exploration of the world by defeasibly assuming that $D \beta$ holds too. Clearly, this is the 
case only if there is no evidence of the contrary - that is, that $D \neg \beta$ holds. As we will see in Section 5, such contrary is formally handled by interaction of inferences in a dialogue, instead of assuming negation as failure $\sim D \neg \beta$ meaning that each attempt to prove $D \neg \beta$ fails.

Definition 7: Positive backward practical syllogism, denoted by PBPS, and negative backward practical syllogism, denoted by NBPS, are defined as follows:

$$
\begin{aligned}
& \text { PBPS : D } \alpha, \gamma, \alpha \wedge \gamma \Rightarrow \beta \rightsquigarrow D \beta ; \\
& \text { NBPS : D } \alpha, \gamma, \neg \alpha \wedge \gamma \Rightarrow \beta \rightsquigarrow D \neg \beta .
\end{aligned}
$$

The positive backward practical syllogism intuitively states that if one believes that $\alpha$ is desirable (e.g. 'cmte democratic' $d$ ), $\gamma$ is the case (e.g. 'the resources committee makes policy about resources' $m$ ) and if $\alpha$ is realised under $\gamma$ is the case then $\beta$ is realised (e.g. 'fairness' $f$ ). Then one defeasibly concludes that $\beta$ might be desirable $(D d, m, d \wedge m \Rightarrow f \rightsquigarrow D f)$. The negative one, however, concludes that $\beta$ is undesirable from a different premise stating that if $\alpha$ is not realised under $\gamma$ is the case then $\beta$ is realised. We describe them as BPS without distinction.

An application of BPS sometimes derives a false conclusion. The following example shows that how such false conclusions can be withdrawn by an interaction of BPS.

Example 1 (continued): The following is an application of a backward practical syllogism:

$$
D d, m, d \wedge m \Rightarrow \neg e \rightsquigarrow D \neg e .
$$

Namely, one believes that a democratic resources committee is desirable (i.e. $D d$ ), the resources committee makes policy about resources (i.e. $m$ ) and if $d$ isrealised under $m$ is the case then it demotes efficiency of the policy (i.e.d $\wedge m \Rightarrow \neg e$ ). From these beliefs, the backward practical syllogism defeasibly derives the belief that an ineffective policy is desirable (i.e. $D \neg e$ ).

This is intuitively a false conclusion. As we will see in Section 5, the conclusion is withdrawn by agent's belief $D e$ or the following another application of a backward practical syllogism:

$$
D e c, m, e c \wedge m \Rightarrow e \rightsquigarrow D e .
$$

Namely, one believes that an efficient resources committee is desirable (i.e.Dec), the resources committee makes policy about resources (i.e. $m$ ) and if $e c$ isrealised under $m$ is the case then it promotes efficiency of the policy (i.e.ec $\wedge m \rightsquigarrow e$ ). From these beliefs, the backward practical syllogism defeasibly derives the belief that an effective policy is desirable.

Finally, in this section, we show the fact that backward practical syllogisms redefine superordinate desires originally defined by forward practical syllogisms.

Proposition 1: Let $D g, D h \in \mathcal{L}_{0}$. Dh is a superordinate desire of $D g$ in $T$ iff there is $\Sigma_{1} \subseteq T$ such that $\Sigma_{1} \cup\{D g\} \vdash_{B P S} D h$ and there is no $\Sigma_{2} \subseteq T$ such that $\Sigma_{2} \cup\{D t\} \vdash_{B P S} D \neg h$, for all $D t \in T \cup\{D g\}$.

\section{Formal protocols of deliberation dialogues}

\subsection{General elements of dialogues}

This section aims to formalise three types of dialogues: a backward deliberation dialogue, a forward deliberation dialogue, and a mixed deliberation dialogue. Particularly, this section gives a 
formal definition of the intersection of backward and forward deliberation dialogues, as general as possible. In general, there are various ingredients associated with formal dialogues, Foe example, locution, reply, commitment, turntaking, termination, and outcomes. In this paper, we only consider locutions, reply, and outcomes because we think that they are necessary and sufficient factors to cover a key part of reconciliation typified by Kowalski and Toni's reconciliatory story. A general framework of reconciliatory dialogues equipped with all of the ingredients is beyond the scope of this paper although it is true that they make reconciliatory dialogues more realistic and sound. In our dialogue setting, unspecified number of players exchange moves during dialogues in which they always have their turn to put forward moves, they are not distinguished from a proponent and opponent, and their locutions are not subject to consistency check with their commitments.

Each move in dialogues consists of a speech act - the content of the move - and a type of dialogue - the context in which moves are put forward.

Definition 8: Let $a \in \mathcal{L}_{0} \cup \mathcal{L}_{1}$. A move is a tuple 〈speechact,type〉 where speech act $\in$ $\{\operatorname{claim}(D \alpha)$, why $(\alpha)$, since $(\Phi \rightsquigarrow \alpha)$, fact $(\alpha)\}$ and type $\in\{B, F\}$, that is, backward deliberation dialogue or forward deliberation dialogue.

Let us define the set of allowed replies to a move.

Definition 9: Let $M$ be a set of moves and $X \in\{B, F\}$. The following table depicts allowed replies to each locution.

\begin{tabular}{ll} 
Locutions & Replies \\
\hline$\langle\operatorname{claim}(D \alpha), X\rangle$ & $\langle\operatorname{why}(\operatorname{D} \alpha), X\rangle,\langle\operatorname{claim}(D \neg \alpha), X\rangle$ \\
$\langle\operatorname{why}(\alpha), X\rangle$ & $\langle\operatorname{since}(\Phi \rightsquigarrow \alpha), X\rangle,\langle\operatorname{fact}(\alpha), X\rangle$ \\
$\langle\operatorname{since}(\Phi=\{\ldots, \beta, \ldots\} \rightsquigarrow \alpha), X\rangle$ & $\langle\operatorname{why}(\beta), X\rangle$ \\
$\langle\operatorname{fact}(\alpha), X\rangle$ &
\end{tabular}

In the following, if $m \in M$ is a reply to $n \in M$, then we will say that $m$ attacks $n$ or $m \longrightarrow n$. Particularly, if $n=\langle\operatorname{claim}(D \alpha), X\rangle$ and $m=\langle\operatorname{claim}(D \neg \alpha), X\rangle$, then $m \longrightarrow n$ and $n \longrightarrow m$. No move attacks a set of moves nor another move with a different type.

A dialogue as a network of moves is defined using a dialogue framework. It is an abstract argumentation framework whose arguments and attacks are instantiated, respectively, by moves and attacks on the set of moves.

Definition 10: A dialogue framework is a pair $D F=\langle M$, attacks $\rangle$, where $M$ is a set of moves and attacks $=\{\langle m, n\rangle \mid m, n \in M, m \longrightarrow n\}$.

Note that dialogue frameworks do not preserve the order in which agents put forward locutions, but preserve only the replying relation between moves. Agents dynamically construct a network of moves, that is, a dialogue framework, by replying to a preceding move in it.

Dung's acceptability semantics evaluates acceptability of moves in dialogue frameworks. This is because it is rational to think that moves successfully replying to critical questions are worthy of acceptance. In general, dialogue frameworks are constructed by multiple agents who freely participate and make moves from their private knowledge bases. This knowledge is invisible to others. They can only see what they said during a dialogue. 


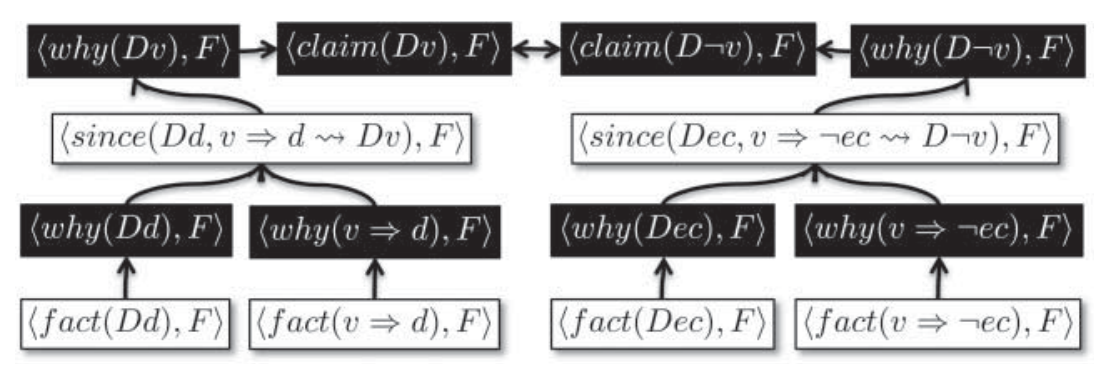

Figure 4. Forward deliberation dialogue with the overruled subject $\langle\operatorname{claim}(D v), F\rangle$ where nodes and links, respectively, represent moves and an attack relation.

Definition 11: Let DF be a dialogue framework. A collaborative theory built from $D F$, denoted by $T(D F)$, is the set $\left\{a \in \mathcal{L}_{0} \cup \mathcal{L}_{1} \mid\right.$ There is a move $m$ in $D F$ whose speech act is fact $\left.(a)\right\}$.

\subsection{Forward and backward deliberation dialogues}

A forward deliberation protocol is defined using forward practical syllogisms.

Definition 12: Let $M$ be a set of moves and $\mathcal{D} \mathcal{F}$ be a set of dialogue frameworks and $D h \in \mathcal{L}_{0} . A$ forward deliberation dialogue protocol is a function $P_{F}: \mathcal{D F} \rightarrow 2^{M}$ where $m \in P_{F}(D F)$ if and only if the following hold:

- $m=\langle\operatorname{claim}(D h), F\rangle$ if $D F=\emptyset$.

- $m \notin D F$ and $\exists n \in D F$ such that $m \longrightarrow n$ if $D F \neq \emptyset$.

Moreover, if the speech act of $m$ is since $(A \rightsquigarrow a)$, then $A \rightsquigarrow a$ is an application of forward practical syllogisms.

A dialogue framework $D F$ is a forward deliberation dialogue iff $D F$ is constructed by the forward deliberation protocol. The first move of $D F$ is called the subject of the dialogue.

Example 1 (continued): Figure 4 presents an example of a forward deliberation dialogue with overruled subject $\langle\operatorname{claim}(D v), F\rangle$ in which white moves are justified and black ones are overruled. The collaborative theory is $T(D F)=\{D d, v \Rightarrow d, D e c, v \Rightarrow \neg e c\}$.

A backward deliberation protocol is defined similarly.

Definition 13: Let $M$ be a set of moves, $\mathcal{D} \mathcal{F}$ be a set of dialogue frameworks, and $D h \in \mathcal{L}_{0}$. $A$ backward deliberation protocol is a function $P_{B}: \mathcal{D F} \rightarrow 2^{M}$ where $m \in P_{B}(D F)$ if and only if the following hold:

- $m=\langle\operatorname{claim}(D h), B\rangle$ if $D F=\varnothing$.

- $m \notin D F$ and $\exists n \in D F$ such that $m \longrightarrow n$ if $D F \neq \emptyset$.

Moreover, if the speech act of $m$ is since $(A \rightsquigarrow a)$, then $A \rightsquigarrow a$ is an application of backward practical syllogisms.

Definition 13 says that the backward deliberation protocol restricts a type of dialogue to a backward deliberation and an inference to backward practical syllogisms. We say that a dialogue framework $D F$ is a backward deliberation dialogue iff $D F$ is constructed by the backward deliberation protocol. 


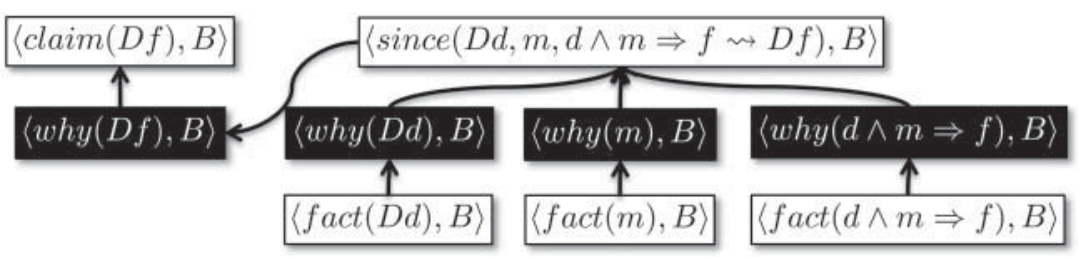

Figure 5. Backward deliberation dialogue with the justified subject $\langle\operatorname{claim}(D f), B\rangle$.

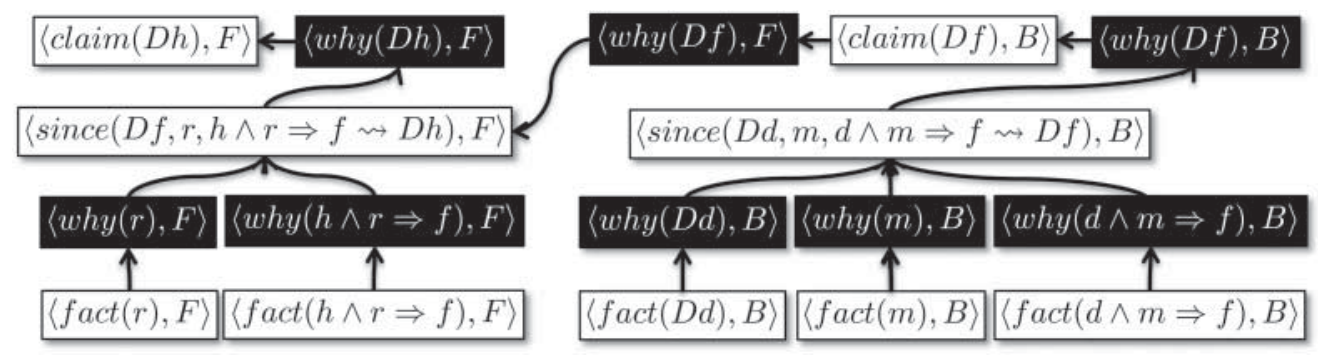

Figure 6. Mixed deliberation dialogue with the justified subject $\langle\operatorname{claim}(D h), F\rangle$.

Example 1 (continued): Figure 5 presents an example of a backward deliberation dialogue in which subject $\langle\operatorname{claim}(D f), B\rangle$ is justified. The collaborative theory is $T(D F)=\{D d, m$, $d \wedge m \Rightarrow f\}$.

\subsection{Mixed deliberation dialogues}

We now define mixed deliberation dialogues by which agents search for and justify reconciliatory desires. We extend the forward and backward protocols so that claim moves in forward deliberation dialogues serve as a trigger to shift from backward to forward deliberation dialogues. Formally, a mixed deliberation protocol allows agents to put forward $\langle\operatorname{claim}(D \alpha), B\rangle$ as a reply to $\langle w h y(D \alpha), F\rangle$ in mixed deliberation dialogues. It is defined based on the forward and backward deliberation dialogue protocols.

Definition 14: Let $N$ be a set of moves and $\mathcal{D} \mathcal{F}$ be a set of dialogue frameworks. A mixed deliberation protocol is a function $P_{M}: \mathcal{D F} \rightarrow 2^{N}$, where $m \in P_{M}(D F)$ iff $m \in P_{F}(D F) \cup P_{B}(D F)$ or $m=\langle\operatorname{claim}(D a), B\rangle$ and $\langle$ why $(D a), F\rangle$ is in $D F$.

A dialogue framework $D F$ is a mixed deliberation dialogue iff $D F$ is constructed using the mixed deliberation protocol. Note that forward and backward deliberation dialogues are both special cases of mixed deliberation dialogues.

Example 1 (continued): Figure 6 presents an example of a mixed deliberation dialogue $D F$ in which the subject $\operatorname{claim}(D h)$ is justified. The collaborative theory built from $D F$ is $T(D F)=$ $\{D d, m, d \wedge m \Rightarrow f, r, h \wedge r \Rightarrow f\}$. 


\section{Correctness of forward, backward, and mixed deliberation dialogues}

\subsection{Forward deliberation dialogues for justifying subordinate desires}

This section shows the relations between acceptability status of dialogue subjects and desires of three kinds (subordinate, superordinate, and reconciliatory desires) defined on collaborative theories. It gives dialogue agents rationale for accepting and agreeing to dialogue subjects.

We say a dialogue framework is finite if the number of moves in it is finite. We impose closedness on dialogue frameworks to associate status of subjects and subordinate, superordinate, and reconciliatory desires. A dialogue framework is closed if it is finite and the corresponding dialogue protocol does not permit to put why and since moves forward.

Definition 15: Let DF be a finite dialogue framework and $P$ be a protocol. DF is closed iff there is no $m \in P(D F)$ such that the speech act of $m$ is why $(a)$, or it is since $(A \rightsquigarrow a)$ where, for all $c \in A$, there is a move $n$ in $D F$ such that the speech act of $n$ is since $(B \rightsquigarrow b)$ and $c \in B$.

We show that subjects of forward deliberation dialogues interpret their dialogue goals, that is, subordinate desires, defined on collaborative theories built through the dialogues. The following two lemmas guarantee that forward deliberation dialogues are sound in the sense that justified subjects are necessarily subordinate desires in the collaborative theory, and complete in the sense that subordinate desires in the collaborative theory are necessarily justified subjects.

Lemma 1: Let $D F$ be a closed forward deliberation dialogue for which the subject is $\langle\operatorname{claim}(D h), F\rangle$. If $\langle\operatorname{claim}(D h), F\rangle$ is justified in $D F$, then there is $D g \in T(D F)$ such that Dh is a subordinate desire of $D g$ in $T(D F)$.

The following lemma assures that forward deliberation dialogues are complete in the sense that subordinate desires in the collaborative theory are necessarily justified subjects.

Lemma 2: Let $D F$ be a closed forward deliberation dialogue for which the subject is $\langle\operatorname{claim}(D h), F\rangle$. If there is $D g \in T(D F)$ such that $D h$ is a subordinate desire of $D g$ in $T(D F)$ then $\langle\operatorname{claim}(D h), F\rangle$ is justified in $D F$.

Lemmas 1 and 2 imply Theorem 1.

Theorem 1: Let DF be a closed forward deliberation dialogue whose subject is $\langle$ claim $(D h), F\rangle$. $\langle\operatorname{claim}(D h), F\rangle$ is justified in DF iff there is $D g \in T(D F)$ such that Dh is a subordinate desire of $D g$ in $T(D F)$.

\subsection{Backward deliberation dialogues for justifying superordinate desires}

Subject status of backward deliberation dialogues interpret their dialogue goals, that is, superordinate desires, defined on collaborative theories built through the dialogues. The following theorem can be shown similarly to Theorem 1 .

Theorem 2: Let DF be a closed backward deliberation dialogue for which the subject is $\langle\operatorname{claim}(D h), B\rangle$. Actually, $\langle$ claim $(D h), B\rangle$ is justified in DF iff there is $D g \in T(D F)$ such that Dh is a superordinate desire of $D g$ in $T(D F)$. 


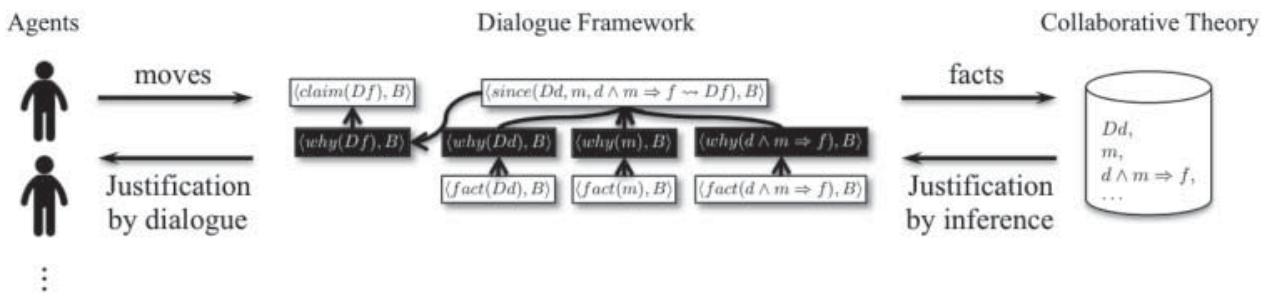

Figure 7. System overview of mixed deliberation dialogue.

\subsection{Mixed deliberation dialogues for justifying reconciliatory desires}

Subject status of mixed deliberation dialogues interpret their dialogue goals, that is, reconciliatory desires, defined on collaborative theories built through the dialogues. The following lemma guarantees that mixed deliberation dialogues are sound in the sense that justified subjects are necessarily reconciliatory desires in collaborative theories.

Lemma 3: Let $X \in\{B, F\}$ and $D F$ be a closed mixed deliberation dialogue for which the subject is $\langle\operatorname{claim}(D h), X\rangle$. If $\langle\operatorname{claim}(D h), X\rangle$ is justified in DF then there is $D g \in T(D F)$ such that Dh is a reconciliatory desire of $D g$ in $T(D F)$.

Lemma 4: Let $X \in\{F, B\}$ and $D F$ be a closed mixed deliberation dialogue for which the subject is $\langle$ claim $(D h), X\rangle$. If $D g \in T(D F)$ exists such that Dh is a reconciliatory desire of $D g$ in $T(D F)$, then $\langle\operatorname{claim}(D h), X\rangle$ is justified in $D F$.

Lemmas 3 and 4 imply Theorem 3.

Theorem 3: Let $X \in\{F, B\}$ and $D F$ be a closed mixed deliberation dialogue for which the subject is $\langle$ claim $(D h), X\rangle$. There is $D g \in T(D F)$ such that $D h$ is a reconciliatory desire of $D g$ in $T(D F)$ iff $\langle\operatorname{claim}(D h), X\rangle$ is justified in $D F$.

\subsection{Illustrative example of mixed deliberation dialogues}

This section gives an illustrative example to show how mixed deliberation dialogues unfold by agents. In Figure 7, we show information flows of our dialogue systems where moves from agents constitute a dialogue framework, and facts from the framework constitute a collaborative theory. Reconciliatory (resp. subordinate, superordinate) desires defined in the collaborative theory give a justification to mixed (resp. forward, backward) deliberation dialogues, and justified claims in the dialogue give a justification to multiagent decision-making.

So far, however, we paid no attention to agent models (the leftmost component in Figure 7) such as agent's knowledge base nor agent's dialogue strategy. This is because we focus on dialogue protocols that should be distinguished from them. A dialogue protocol deals with moves agents are allowed to put forward in dialogues. On the other hand, a dialogue strategy deals with moves agents actually put forward in dialogues. Agent's knowledge base defines moves she can make from her knowledge base, and this information affects her strategy of what to say in dialogues. However, it does not affect dialogue protocols of what moves she is allowed to put forward.

However, we think that agents' knowledge and strategies are necessary to show how a dialogue unfold by them. In this section, we assume two agents agent 1 and agent $_{2}$ who have the 
following knowledge bases, $T_{1}, T_{2} \subseteq \mathcal{L}_{0} \cup \mathcal{L}_{1}$, respectively:

$$
\begin{aligned}
& T_{1}=\{D d, v \Rightarrow d, m, r, a \Rightarrow e c\}, \\
& T_{2}=\{D e c, v \Rightarrow e c, d \wedge m \Rightarrow f, h \wedge r \Rightarrow f\} .
\end{aligned}
$$

Moreover, we assume the simple strategy that each agent willingly and honestly participates in dialogues with no particular order. By permission of a given dialogue protocol, each agent puts forward fact and since moves she can make from her knowledge base. She also puts forward why moves in any time and claim moves in the beginning of dialogues.

For example, consider the situation where agent $_{1}$ starts a dialogue on the subject whether $v$ (i.e. 'voting') is a subordinate (and therefore reconciliatory) desire or not.

\begin{tabular}{llll} 
Utterer & Move & Attack-to & Status \\
\hline agent $_{1}$ & $\langle\operatorname{claim}(D v), F\rangle$ & - & overruled \\
agent $_{2}$ & $\langle$ why $(D v), F\rangle$ & $\langle\operatorname{claim}(D v), F\rangle$ & overruled \\
agent $_{1}$ & $\langle\operatorname{since}(D d, v \Rightarrow d \rightsquigarrow D v), F\rangle$ & $\langle$ hhy $(D v), F\rangle$ & justified \\
agent $_{2}$ & $\langle$ why $(D d), F\rangle$ & $\langle\operatorname{since}(D d, v \Rightarrow d \rightsquigarrow D v), F\rangle$ & overruled \\
agent $_{2}$ & $\langle$ why $(v \Rightarrow d), F\rangle$ & $\langle\operatorname{since}(D d, v \Rightarrow d \rightsquigarrow D v), F\rangle$ & overruled \\
agent $_{1}$ & $\langle$ fact $(D d), F\rangle$ & $\langle$ why $(D d), F\rangle$ & justified \\
agent $_{1}$ & $\langle\operatorname{fact}(v \Rightarrow d), F\rangle$ & $\langle$ hhy $(v \Rightarrow d), F\rangle$ & justified \\
agent $_{2}$ & $\langle\operatorname{claim}(D \neg v), F\rangle$ & $\langle\operatorname{claim}(D v), F\rangle$ & overruled \\
agent $_{1}$ & $\langle$ why $(D \neg v), F\rangle$ & $\langle\operatorname{claim}(D \neg v), F\rangle$ & overruled \\
agent $_{2}$ & $\langle\operatorname{since}(D e c, v \Rightarrow \neg e c \rightsquigarrow D \neg v), F\rangle$ & $\langle$ why $(D \neg v), F\rangle$ & justified \\
agent $_{1}$ & $\langle$ why $(D e c), F\rangle$ & $\langle\operatorname{since}(D e c, v \Rightarrow \neg e c \rightsquigarrow D \neg v), F\rangle$ & overruled \\
agent $_{1}$ & $\langle$ why $(v \Rightarrow \neg e c), F\rangle$ & $\langle\operatorname{since}(D e c, v \Rightarrow \neg e c \rightsquigarrow D \neg v), F\rangle$ & overruled \\
agent $_{2}$ & $\langle\operatorname{fact}(D e c), F\rangle$ & $\langle$ hhy $(D e c), F\rangle$ & justified \\
agent $_{2}$ & $\langle\operatorname{fact}(v \Rightarrow \neg e c), F\rangle$ & $\langle$ hhy $(v \Rightarrow \neg e c), F\rangle$ & justified
\end{tabular}

The above table shows a sequence of moves and their utterers, targets, and status in the forward (and therefore mixed) deliberation dialogue where the subject $\langle\operatorname{claim}(D v), F\rangle$ is overruled. Now, consider the another situation where agent ${ }_{1}$ starts another dialogue on the subject whether

\begin{tabular}{|c|c|c|c|}
\hline Utterer & Move & Attack-to & Status \\
\hline agent $_{2}$ & $\langle\operatorname{claim}(D h), F\rangle$ & - & justified \\
\hline agent $_{1}$ & $\langle w h y(D h), F\rangle$ & $\langle\operatorname{claim}(D h), F\rangle$ & overruled \\
\hline agent $_{2}$ & $\langle\operatorname{since}(D f, r, h \wedge r \Rightarrow f \rightsquigarrow D h), F\rangle$ & $\langle w h y(D h), F\rangle$ & justified \\
\hline agent $_{1}$ & $\langle w h y(D f), F\rangle$ & $\langle\operatorname{since}(D f, r, h \wedge r \Rightarrow f \rightsquigarrow D h), F\rangle$ & overruled \\
\hline agent $_{1}$ & $\langle w h y(h \wedge r \Rightarrow f), F\rangle$ & $\langle\operatorname{since}(D f, r, h \wedge r \Rightarrow f \rightsquigarrow D h), F\rangle$ & overruled \\
\hline agent $_{1}$ & $\langle w h y(r), F\rangle$ & $\langle\operatorname{since}(D f, r, h \wedge r \Rightarrow f \rightsquigarrow D h), F\rangle$ & overruled \\
\hline agent $_{2}$ & $\langle\operatorname{claim}(D f), B\rangle$ & $\langle w h y(D f), F\rangle$ & justified \\
\hline agent $_{2}$ & $\langle$ fact $(h \wedge r \Rightarrow f), F\rangle$ & $\langle w h y(h \wedge r \Rightarrow f), F\rangle$ & justified \\
\hline agent $_{1}$ & $\langle$ fact $(r), F\rangle$ & $\langle w h y(r), F\rangle$ & justified \\
\hline agent $_{1}$ & $\langle w h y(D f), B\rangle$ & $\langle\operatorname{claim}(D f), B\rangle$ & overruled \\
\hline agent $_{2}$ & $\langle\operatorname{since}(D d, m, d \wedge m \Rightarrow f \rightsquigarrow D f), B\rangle$ & $\langle w h y(D f), B\rangle$ & justified \\
\hline agent $_{1}$ & $\langle w h y(D d), B\rangle$ & $\langle\operatorname{since}(D d, m, d \wedge m \Rightarrow f \rightsquigarrow D f), B\rangle$ & overruled \\
\hline agent $_{1}$ & $\langle w h y(m), B\rangle$ & $\langle\operatorname{since}(D d, m, d \wedge m \Rightarrow f \rightsquigarrow D f), B\rangle$ & overruled \\
\hline agent $_{1}$ & $\langle w h y(d \wedge m \Rightarrow f), B\rangle$ & $\langle\operatorname{since}(D d, m, d \wedge m \Rightarrow f \rightsquigarrow D f), B\rangle$ & overruled \\
\hline agent $_{1}$ & $\langle\operatorname{fact}(D d), B\rangle$ & $\langle w h y(D d), B\rangle$ & justified \\
\hline agent $_{1}$ & $\langle\operatorname{fact}(m), B\rangle$ & $\langle w h y(m), B\rangle$ & justified \\
\hline agent $_{2}$ & $\langle\operatorname{fact}(d \wedge m \Rightarrow f), B\rangle$ & $\langle w h y(d \wedge m \Rightarrow f), B\rangle$ & justified \\
\hline
\end{tabular}
$h$ (i.e. 'head cmte administrative only') is a reconciliatory desire or not. 
The above table shows a sequence of moves and their utterers, targets, and status in the mixed deliberation dialogue where the subject $\langle\operatorname{claim}(D h), F\rangle$ is justified.

We can also see that the subject $\langle\operatorname{claim}(D a), F\rangle$ is justified in the following forward (and therefore mixed deliberation) dialogue started by agent $t_{2}$. Note that each reconciliatory desire justified in dialogues is derived neither from $T_{1}$ nor $T_{2}$ using forward and backward practical syllogisms.

\begin{tabular}{llll} 
Utterer & Move & Attack-to & Status \\
\hline agent $_{2}$ & $\langle\operatorname{claim}(D a), F\rangle$ & - & justified \\
agent $_{1}$ & $\langle$ why $(D a), F\rangle$ & $\langle\operatorname{claim}(D a), F\rangle$ & overruled \\
agent $_{1}$ & $\langle\operatorname{since}(\operatorname{Dec}, a \Rightarrow e c \rightsquigarrow D a), F\rangle$ & $\langle$ why $(\operatorname{Da}), F\rangle$ & justified \\
agent $_{2}$ & $\langle$ why $(\operatorname{Dec}), F\rangle$ & $\langle\operatorname{since}(\operatorname{Dec}, a \Rightarrow e c \rightsquigarrow D a), F\rangle$ & overruled \\
agent $_{2}$ & $\langle$ why $(a \Rightarrow e c), F\rangle$ & $\langle\operatorname{since}(\operatorname{Dec}, a \Rightarrow e c \rightsquigarrow D a), F\rangle$ & overruled \\
agent $_{2}$ & $\langle$ fact $(\operatorname{Dec}), F\rangle$ & $\langle$ why $(\operatorname{Dec}), F\rangle$ & justified \\
agent $_{1}$ & $\langle$ fact $(a \Rightarrow e c), F\rangle$ & $\langle$ why $(a \Rightarrow e c), F\rangle$ & justified
\end{tabular}

\section{Related work and discussions}

Focusing on superordinate or underlying desires furthers reconciliation. Fisher, Ury, and Patton (1992) argue that paying attention to stakeholders' interests helps to find reconciliation in negotiation. Brett (2014) says that negotiation theory distinguishes a position and an interest where a proposition is what negotiators say they want and an interest is the needs of concerns that underlie positions.

In argument-based negotiation and deliberation, the research studies (Amgoud, Devred, and Lagasquie-Schiex, 2009; Hulstijn and van der Torre, 2004; Modgil and Luck, 2009) use practical reasoning to deal with desire derivation and generation mechanisms for knowledgedependent and context-dependent desires. They, however, do not address the situations in which there is no means to achieve original nor derivative desires. Rahwan, Pasquier, Sonenberg, and Dignum (2007) argue that underlying goals improve negotiation processes and consider desires hierarchised in advance. In contrast to their approach, we assume the situation where desires are structurised as a result of inference by forward and backward practical syllogisms. Our approach is necessary when agents have incomplete desires, as well as incomplete knowledge. Hitchcock, McBurney, and Parsons (2001) and McBurney, Hitchcock, and Parsons (2007) propose deliberation dialogue frameworks, for example, DDF (McBurney et al., 2007), equipped with fundamental elements for deliberation dialogues such as locutions, commitments, and termination. Kok et al. (2010) give an argumentation framework for deliberation dialogue taking into account agent's preference. However, these frameworks do not address evaluation of their correctness. In this paper, we gave proof-based evaluation for correctness of our dialogues in terms of dialogue goals. Fan and Toni (2012) use an assumption-based argumentation framework to relate successful dialogues with admissible arguments in the framework. Wells and Reed (2006) handle a shift from persuasion to negotiation, and demonstrate its effectiveness using an example. Although we also use argumentation frameworks and dialectical shifts to define our dialogues, the type of dialogue and the goal of dialogue, we focus on, are uniquely deliberation and reconciliatory, respectively.

Kido and Ohsawa (2013) propose a reconciliatory argumentation system instantiating Dung's argumentation framework with modal propositional language and rules of practical inference. Our dialogue-based reconciliation has an advantage over argumentation-based in the sense that it 
handles circumstances under which individual agents cannot make a reconciliatory argument by themselves because of lack of knowledge, but can make such an argument for mutual coverage. Moreover, dialogue-based approaches succeed in capturing dynamic aspects of interaction where individual agents build a theory collaboratively during a dialogue. On the other hand, this paper has the limitation that dialogue protocols allow agents to use only practical syllogisms and allow them to attack only moves except facts. We think that our work can be extended to a general reconciliatory dialogue by utilising the persuasion dialogues mentioned above and inquiry dialogues (Black and Hunter, 2009) allowing agents to share knowledge to jointly construct arguments or dialectical trees.

Much work for formal deliberation dialogues has been inspired by studies of formal persuasion dialogues. Prakken (2005) provides a formal dialogue system and shows that, under some conditions, a proponent wins in a dialogue iff a topic of the dialogue is defeasibly derived from agreed information. We also adopt a similar manner in giving the correctness of our dialogue protocols because we agree with Carlson's idea (1983), summarized by Prakken (2005, 2006), that

whereas logic defines the conditions under which a proposition is true, dialogue systems define the conditions under which an utterance is appropriate, and this is the case if the utterance furthers the goal of the dialogue in which it is made.

However, our dialogue protocols are completely different to Prakken's because of differences of dialogue types and goals. It is true that there are various ingredients associated with formal dialogues, for example, locution, reply, commitment, turntaking, termination, and outcomes, and our protocols deal with some of them, in contrast to Prakken's protocols. However, our approach does not conflict with his approach because, as Prakken mentioned in Prakken (2005), his structure of dialogues is especially suited for 'verbal struggles' and he does not claim that all dialogues should or do conform to the structure. In fact, our dialogues have little interest in commitment because consistency evaluation of locutions in terms of commitment does not directly contribute to the solution of Kowalski and Toni's scenario. This is supported by the fact that Kowalski and Toni's scenario makes no mention of agent's commitment in the process of reaching the reconciliatory solution. Our dialogues neither have an interest in an wining-or-losing criterion because agents do not compete against each other with the different roles, a proponent and an opponent, but collaborate each other to find a reconciliation. Turntaking is neither an essential because agents having the same role do not need to be distinguished. A termination criterion is not essential because Dung's grounded semantics allows us to give outcomes of any particular situation of dialogues. It is true that all of these factors make our dialogues more realistic and sound. However, pursuing a generality of reconciliatory dialogues is beyond the scope of this paper.

\section{Conclusions and future work}

We proposed a mixed deliberation dialogue for reconciliatory desires. A mixed deliberation dialogue is defined as a combination of forward and backward deliberation dialogues for which the goals are subordinate and superordinate desires, respectively. We showed the correctness of dialogues based on the fact that the subject of closed mixed deliberation dialogue is justified iff the subject is a reconciliatory desire of a desire in the collaborative theory, that is, iff the dialogue satisfies its goal. Weaknesses of our formalisation are, first, that it restricts inference mechanisms to only forward and backward practical syllogisms, and second, that it does not allow agents to challenge or rebut facts put forward in dialogues. We will utilise existing persuasion and inquiry dialogues to address these issues. 


\section{Disclosure statement}

No potential conflict of interest was reported by the authors.

\section{Funding}

This research was partially supported by the Telecommunication Advancement Foundation, Japan.

\section{Note}

1. The authors originally call them positive and negative practical syllogisms, respectively, and the conclusion part of the negative one is $\neg D \beta$.

\section{References}

Amgoud, L., Devred, C., \& Lagasquie-Schiex, M. (2009). A constrained argumentation system for practical reasoning. In Proceedings of the fifth international workshop on argumentation in multi-agent systems, Estoril, Portugal (pp. 37-56).

Bench-Capon, T. J. M., \& Prakken., H. (2006). Justifying actions by accruing arguments. In Proceedings of the 1st international conference on computational models of argument, Liverpool, UK (pp. 247-258).

Black, E., \& Hunter, A. (2009). An inquiry dialogue system. JAAMAS, 19, 173-209.

Brett, J. M. (2014). Negotiating globally: How to negotiate deals, resolve disputes, and make decisions across cultural boundaries (Jossey-Bass Business \& Management) (3rd ed.). Jossey-Bass, San Francisco, CA, USA.

Carlson, L. (1983). Dialogue games: An approach to discourse analysis. Dordrecht: Reidel Publishing.

Dung, P. M. (1995). On the acceptability of arguments and its fundamental role in nonmonotonic reasoning, logic programming, and $n$-person games. Artificial Intelligence, 77, 321-357.

van Eemeren, F. H., Grootendorst, R., \& Henkemans, F. S. (1996). Fundamentals of argumentation theory: A handbook of historical backgrounds and contemporary developments. Routledge.

Fan, X., \& Toni, F. (2012). Argumentation dialogues for two-agent conflict resolution. In Proceedings of the 4th international conference on computational models of argument, Vienna, Austria (pp. 249-260).

Fisher, R., Ury, W., \& Patton, B. (1992). Getting to yes: Negotiating agreement without giving in (2nd ed.). Houghton Mifflin Harcourt, New York, USA.

Hamblin, C. L. (1970). Fallacies. Methuen, London, UK.

Hintikka, J. (1968). Language-games for quantifiers (pp. 46-72). Oxford: Blackwell, in American Philosophical Quarterly Monograph Series 2: Studies in Logical Theory Edition.

Hitchcock, D., McBurney, P., \& Parsons, S. (2001). A framework for deliberation dialogues. In Proceedings of the 4th biennial conference of the Ontario Society for the Study of Argumentation, Windsor, Ontario, Canada (pp. 1-24).

Hulstijn, J., \& van der Torre, L. (2004). Combining goal generation and planning in an argumentation framework. In Proceedings of the 10th international workshop on non-monotonic reasoning, Whistler, BC, Canada (pp. 212-218).

Kido, H., \& Cerutti, F. (2014). Shift from forward to backward deliberation in search of reconciliation. In Proceedings of the 13th Pacific Rim International Conference on Artificial Intelligence (PRICAI 2014), Gold Coast, Queensland, Australia (pp. 920-928).

Kido, H., \& Ohsawa, Y. (2013). Justifying underlying desires for argument-based reconciliation. In Proceedings of the 2nd international workshop on Theory and Applications of Formal Argumentation (TAFA 2013), Beijing, China (pp. 143-157).

Kok, E. M., Meyer, J. J. C., Prakken, H., \& Vreeswijk, G. A. W. (2010). A formal argumentation framework for deliberation dialogues. In Proceedings of the 7 th international workshop on argumentation in multiagent systems, Toronto, Canada (pp. 31-48). 
Kowalski, R. A., \& Toni, F. (1994). Argument and reconciliation. In Proceedings of the 5th generation computer systems workshop on application of logic programming to legal reasoning, Tokyo, Japan (pp. 9-16).

Lorenzen, P. (1961). Ein dialogisches konstruktivittskriterium (pp. 193-200). Oxford: Pergamon, in Infinitistic Methods Edition.

McBurney, P., Hitchcock, D., \& Parsons, S. (2007). The eightfold way of deliberation dialogue. International Journal of Intelligent Systems, 22(1), 95-132.

Modgil, S., \& Luck, M. (2009). Argumentation based resolution of conflicts between desires and normative goals. In Proceedings of the 5th international workshop on Argumentation in Multi-Agent Systems (ArgMAS 2009), Estoril, Portugal (pp. 19-36).

Prakken, H. (2005). Coherence and flexibility in dialogue games for argumentation. Journal of Logic and Computation, 15, 1009-1040.

Prakken, H. (2006). Formal systems for persuasion dialogue. The Knowledge Engineering Review, 21(2), 163-188.

Rahwan, I., Pasquier, P., Sonenberg, L., \& Dignum, F. (2007). On the benefits of exploiting underlying goals in argument-based negotiation. In Proceedings of the 22nd national conference on artificial intelligence (AAAI 2007), Vancouver, BC, Canada (pp. 116-121).

Wells, S., \& Reed, C. (2006). Knowing when to bargain. In Proceedings of the 1st international conference on computational models of argument, Liverpool, UK (pp. 235-246).

\section{Appendix. Proofs}

Proposition 1: Let $D g, D h \in \mathcal{L}_{0}$. Dh is a superordinate desire of $D g$ in $T$ iff there is $\Sigma_{1} \subseteq T$ such that $\Sigma_{1} \cup\{D g\} \vdash_{B P S}$ Dh and there is no $\Sigma_{2} \subseteq T$ such that $\Sigma_{2} \cup\{D t\} \vdash_{B P S} D \neg h$, for all $D t \in T \cup\{D g\}$.

Proof: It is obvious that, for any $\Sigma \subseteq T$ and $D x, D y \in \mathcal{L}_{0}, \Sigma \cup\{D x\} \vdash_{F P S} D y$ iff $\Sigma \cup\{D y\} \vdash_{B P S} D x$. So, $D h$ is a superordinate desire of $D g$ in $T$ iff (1) there is $\Sigma_{1} \subseteq T$ such that $\Sigma_{1} \cup\{D g\} \vdash_{B P S} D h$ and (2) there is no $\Sigma_{2} \subseteq T$ such that $\Sigma_{2} \cup\{D t\} \vdash_{B P S} D \neg h$, for all $D t \in T \cup\{D g\}$.

Lemma 1: Let DF be a closed forward deliberation dialogue for which the subject is $\langle\operatorname{claim}(D h), F\rangle$. If $\langle$ claim $(D h), F\rangle$ is justified in $D F$, then there is $D g \in T(D F)$ such that $D h$ is a subordinate desire of $D g$ in $T(D F)$.

Proof: The following proof still holds when $a_{i+1} \wedge b_{i} \Rightarrow a_{i}$ is replaced by $\neg a_{i+1} \wedge b_{i} \Rightarrow \neg a_{i}$. Since $D F$ is closed and $\langle\operatorname{claim}(D h), F\rangle$ is justified, fact $(D h)$ appears in $D F$ or there is $D a_{1} \in \mathcal{L}_{0} \cup \mathcal{L}_{1}$ such that fact $\left(D a_{1}\right)$ appears in $D F$ and, for all $i(1 \leq i \leq n)$, there are $a_{i}, b_{i} \in \mathcal{L}_{0} \cup \mathcal{L}_{1}$ such that fact $\left(b_{i}\right)$, fact $\left(a_{i+1} \wedge b_{i} \Rightarrow a_{i}\right)$, and since $\left(D a_{i}, b_{i}, a_{i+1} \wedge b_{i} \Rightarrow a_{i} \rightsquigarrow D a_{i+1}\right)$ appear in $D F$ where $a_{n+1}=h$. Therefore, $D a_{1} \in T(D F)$ and $b_{i}, a_{i+1} \wedge b_{i} \Rightarrow a_{i} \in T(D F)$, for all $i(1 \leq i \leq n)$.

Based on proof by contradiction, we show there is no $\Sigma \subseteq T(D F)$ such that $\Sigma \cup\{D t\} \vdash_{F P S} D \neg h$, for all $D t \in T(D F) \cup\{D g\}$. If $\Sigma \cup\{D t\} \vdash_{F P S} D \neg h$ then fact $\left(D a_{1}\right)$, fact $\left(b_{i}\right)$, and fact $\left(a_{i+1} \wedge b_{i} \Rightarrow a_{i}\right)$ appear in $D F$, for all $i(1 \leq i \leq n)$, where $a_{n+1}=\neg h$. So, $\left\langle w h y\left(D a_{i}\right), F\right\rangle,\left\langle w h y\left(b_{i}\right), F\right\rangle$, and $\left\langle w h y\left(a_{i+1} \wedge b_{i} \Rightarrow a_{i}\right), F\right\rangle$ are all attacked, for all $i(1 \leq i \leq n)$. Therefore, it is not the case that $\langle\operatorname{claim}(D \neg h), F\rangle$ is overruled. Since $\langle\operatorname{claim}(D \neg h), F\rangle$ attacks $\langle\operatorname{claim}(D h), F\rangle,\langle\operatorname{claim}(D h), F\rangle$ cannot be justified in $D F$.

Lemma 2: Let DF be a closed forward deliberation dialogue for which the subject is $\langle$ claim $(D h), F\rangle$. If there is $D g \in T(D F)$ such that Dh is a subordinate desire of $D g$ in $T(D F)$ then $\langle\operatorname{claim}(D h), F\rangle$ is justified in $D F$.

Proof: The following proof still holds when $a_{i+1} \wedge b_{i} \Rightarrow a_{i}$ is replaced by $\neg a_{i+1} \wedge b_{i} \Rightarrow \neg a_{i}$. There is $\Sigma_{1} \subseteq T(D F)$ such that $\Sigma_{1} \cup\{D g\} \vdash_{F P S} D h$ and there is no $\Sigma_{2} \subseteq T(D F)$ such that $\Sigma_{2} \cup\{D t\} \vdash_{F P S} D \neg h$, for all $D t \in T(D F) \cup\{D g\}$. Since $\langle$ fact $(D g), F\rangle$ appears in $D F$ and there are $a_{i}, b_{i} \in \mathcal{L}_{0} \cup \mathcal{L}_{1}$ such that $\left\langle f a c t\left(b_{i}\right), F\right\rangle$ and $\left\langle\right.$ fact $\left.\left(a_{i+1} \wedge b_{i} \Rightarrow a_{i}\right), F\right\rangle$ appear in $D F$, for all $i(1 \leq i \leq n)$, where $a_{1}=g$ and $a_{n+1}=h$. So, for all $i(1 \leq i \leq n),\left\langle w h y\left(D a_{i}\right), F\right\rangle,\left\langle w h y\left(b_{i}\right), F\right\rangle$, and $\left\langle w h y\left(a_{i+1} \wedge b_{i} \Rightarrow a_{i}\right), F\right\rangle$ are all attacked.

Since there is $i(1 \leq i \leq n)$ such that no moves $\left\langle\operatorname{fact}\left(D c_{1}\right), F\right\rangle$, $\left\langle\operatorname{fact}\left(d_{i}\right), F\right\rangle$, or $\left\langle\operatorname{fact}\left(c_{i+1} \wedge d_{i} \Rightarrow\right.\right.$ $\left.\left.c_{i}\right), F\right\rangle$ appear in $D F$, where $c_{n+1}=\neg h$, there is $i(1 \leq i \leq n)$ such that $\left\langle w h y\left(D c_{i}\right), F\right\rangle,\left\langle w h y\left(d_{i}\right), F\right\rangle$, or 
$\left\langle w h y\left(c_{i+1} \wedge d_{i} \Rightarrow c_{i}\right), F\right\rangle$ is not attacked and justified. Since $\langle\operatorname{claim}(D \neg h), F\rangle$ is overruled, $\langle\operatorname{claim}(D h), F\rangle$ is justified.

Theorem 1: Let DF be a closed forward deliberation dialogue whose subject is $\langle$ claim $(D h), F\rangle$. $\langle\operatorname{claim}(D h), F\rangle$ is justified in DF iff there is $D g \in T(D F)$ such that Dh is a subordinate desire of $D g$ in $T(D F)$.

Proof: Immediate from Lemmas 1 and 2.

Theorem 2: Let DF be a closed backward deliberation dialogue for which the subject is $\langle$ claim $(D h), B\rangle$. Actually, $\langle\operatorname{claim}(D h), B\rangle$ is justified in DF iff there is $D g \in T(D F)$ such that Dh is a superordinate desire of $D g$ in $T(D F)$.

Proof: As a result of Proposition 1, the proof is same as Theorem 1.

Lemma 3: Let $X \in\{B, F\}$ and $D F$ be a closed mixed deliberation dialogue for which the subject is $\langle\operatorname{claim}(D h), X\rangle$. If $\langle\operatorname{claim}(D h), X\rangle$ is justified in DF then there is $D g \in T(D F)$ such that $D h$ is a reconciliatory desire of $D g$ in $T(D F)$.

Proof: It is sufficient to consider a mixed deliberation dialogue $D F$ whose subject $\operatorname{claim}(D h)$ is not justified in neither only forward nor backward deliberation dialogues appeared in $D F$ since otherwise the proof is reduced to Theorems 1 and 2. So, the only situation is that the dialogue starts with a forward deliberation dialogue whose subject $\langle\operatorname{claim}(D h), F\rangle$ is not justified in the dialogue, and then, shifts to backward deliberation dialogues and one, denoted by $D F_{b}$, of them makes it justified in $D F$. The subject, denoted by $\langle\operatorname{claim}(D g), B\rangle$, of $D F_{b}$ is justified because otherwise $D F_{d}$ cannot make $\langle\operatorname{claim}(D h), F\rangle$ justified in $D F$. From Theorem 1, this means that $D g$ is a superordinate desire of a desire in $T(D F)$. Since $\langle\operatorname{claim}(D h), F\rangle$ is justified in $D F$ by the existence of $\operatorname{claim}(D g)$, Theorem 2 implies that $D h$ is a subordinate desire of $D g$ in $T(D F)$.

Lemma 4: Let $X \in\{F, B\}$ and $D F$ be a closed mixed deliberation dialogue for which the subject is $\langle\operatorname{claim}(D h), X\rangle$. If $D g \in T(D F)$ exists such that $D h$ is a reconciliatory desire of $D g$ in $T(D F)$, then $\langle\operatorname{claim}(D h), X\rangle$ is justified in DF.

Proof: It is sufficient to consider $D h$ that is neither subordinate nor superordinate desires of $D g$ because otherwise these cases reduce the proof to Theorems 1 and 2. Let $D i \in \mathcal{L}_{0} \backslash T(D F)$ be a desire such that $D h$ is a subordinate desire of $D i$ and $D i$ is a superordinate desire of $D g$ in $T(D F)$. From Theorem 1, there is a backward deliberation dialogue, denoted by $B D$, whose subject $\langle\operatorname{claim}(D i), B\rangle$ is justified in the dialogue. On the other hand, we can consider the forward deliberation dialogue, denoted by $F D$, whose root is $\langle\operatorname{claim}(D h), F\rangle$ where the root is overruled due to the fact that $\langle w h y(D i), F\rangle$ is justified. However, $\langle\operatorname{claim}(D h), F\rangle$ becomes justified in the mixture of $B D$ and $F D$ because $\langle\operatorname{claim}(D i), B\rangle$ makes $\langle w h y(D i), F\rangle$ overruled.

Theorem 3: Let $X \in\{F, B\}$ and $D F$ be a closed mixed deliberation dialogue for which the subject is $\langle$ claim $(D h), X\rangle$. There is $D g \in T(D F)$ such that $D h$ is a reconciliatory desire of $D g$ in $T(D F)$ iff $\langle\operatorname{claim}(D h), X\rangle$ is justified in $D F$.

Proof: Immediate from Lemmas 3 and 4. 